\section{REASENTAMIENTO POR UN MEGA PROYECTO DE INFRAESTRUCTURA EN LIMA, PERÚ. UN ANÁLISIS DESDE LA HABITABILIDAD Y LAS RELACIONES SOCIALES ${ }^{1}$}

Guillermo Takano

\section{Resumen}

Las dinámicas de reestructuración metropolitana vienen acompañadas de procesos tales como el reasentamiento involuntario de la población, que, si bien se espera se den a cambio de equiparar o mejorar condiciones de vida preexistentes, han tendido a intensificar situaciones de vulnerabilidad. El artículo se enfoca en el reasentamiento generado por el megaproyecto de transporte "Línea Amarilla”, localizado en Lima, Perú. Utilizando métodos cuantitativos y cualitativos se procesó información actual y retrospectiva sobre el reasentamiento en el complejo de vivienda "Patio Unión", con el objetivo de medir las variaciones en las condiciones de las

\section{RESETTLEMENT RESULTING FROM THE DEVELOPMENT OF AN INFRASTRUCTURE MEGAPROJECT IN LIMA, PERU. AN ANALYSIS ON HOUSING HABITABILITY AND SOCIAL RELATIONS ${ }^{1}$}

\author{
Guillermo Takano²
}

\section{Abstract}

Metropolitan restructuring processes are often followed by issues such as involuntary resettlement. Even though it is hoped that such projects will compare with or improve preexisting living conditions, they instead intensified vulnerability. This paper is focused on the resettlement caused by the "Línea Amarilla" transport megaproject carried out in Lima-Peru, specifically around the "Patio Unión" housing complex. Both present and retrospective information were processed by using quantitative and qualitative methods in order to measure variations in housing and household conditions. These variations were related with the 
viviendas y los hogares afectados, relacionándolas con los mecanismos empleados y las decisiones tomadas. Se muestra que ante un esquema de compensación percibido como injusto, los afectados optaron por trasladarse a Patio Unión para permanecer cerca de sus barrios y no perder sus redes de subsistencia económica. Sin embargo, ello se dio a cambio de la pérdida de habitabilidad, el desmembramiento de las familias extendidas, el desgaste del tejido social y la pérdida de la vivienda como medio de producción e inversión. El estudio concluye que el alcance de los mecanismos de reasentamiento y compensación debe expandirse, de modo que se capture la complejidad de la vivienda como un activo familiar fundamental.

\section{PALABRAS CLAVE: REASENTAMIENTO INVOLUNTARIO; DIDR; SUSTENTO FAMILIAR; VIVIENDA SOCIAL.}

\section{Recibido: 06-03-2018}

Aceptado: 27-08-2018

1 La elaboración del estudio fue enteramente financiada por el Instituto de Investigación Científica (IDIC) de la Universidad de Lima.

2 Perú. Profesor e investigador de la Facultad de Ingeniería y Arquitectura de la Universidad de Lima. ORCID: http://orcid. org/0000-0002-3186-8676. Correo electrónico: Itakano@ulima. edu.pe. mechanisms and decisions that led the process. We found that affected populations chose to move to Patio Unión in order to stay close to their neighborhoods and maintain their economic subsistence networks. Nevertheless, the latter happened at the cost of losing conditions of habitability, broke apart extended families, undermining the social tissue and losing the investment and productive values of dwellings. The study concludes that the scope of resettlement and compensation mechanisms should expand in order to capture the complexity of housing as a fundamental asset.

\section{KEYWORDS: INVOLUNTARY RESETTLEMENT; DIDR; FAMILY LIVELIHOODS; SOCIAL HOUSING.}

Received: 06-03-2018

Accepted: 27-08-2018

1 The present paper was entirely financed by the Institute for Scientific Research (IDIC), University of Lima

2 Peru. Professor and researcher, Faculty of Engineering and Architecture, University of Lima. ORCID: http://orcid.org/00000002-3186-8676. Email: Itakano@ulima.edu.pe. 


\section{Introducción}

En el actual contexto de globalización las ciudades se vienen consolidando como motores del crecimiento económico, y, en consecuencia, transformando. Ello también incorpora a las ciudades del Sur Global, muchas de ellas marcadas históricamente por la pobreza, y donde los sectores marginados terminan cubriendo muchos de los costos (Robinson, 2003), por ejemplo, mediante procesos de reasentamiento involuntario que, si bien se espera que equiparen o mejoren condiciones previas de vida, tienden más bien a intensificar la vulnerabilidad de las personas.

Se busca estudiar el proceso de reasentamiento de los afectados por el megaproyecto de transporte "Línea Amarilla" localizado en Lima, Perú, tomando como caso de estudio el complejo de vivienda "Patio Unión". Se parte de tres preguntas clave: (1) ¿cuáles son las condiciones espaciales y socioeconómicas de los hogares comparadas con las condiciones previas al reasentamiento?, (2) ¿cuál es la relación entre las preferencias de los hogares y las variaciones encontradas? y (3) ¿hasta qué punto es posible asociar los hallazgos con el diseño y desempeño del proceso de reasentamiento?

El estudio está asociado a la literatura sobre "Desplazamiento y reasentamiento inducido por el desarrollo" (DIDR). Esperamos aportar a dicho cuerpo de conocimiento con aspectos empíricos y conceptuales sobre cómo se produce y reproduce la vivienda y la ciudad en el contexto latinoamericano, así como generar experiencia de caso relevante para la discusión general sobre políticas de vivienda social en contextos de reasentamiento involuntario. El artículo está estructurado de la siguiente manera: la primera sección presenta una revisión de la literatura; la siguiente sección presenta la metodología y a continuación se presenta el caso de estudio y los hallazgos, los cuales se discuten en la sección siguiente. Finalmente, se presentan las conclusiones y recomendaciones en la última sección.

\section{Revisión de la literatura}

La ola de modernización económica dada después de la Segunda Guerra Mundial tuvo al desarrollo de infraestructura como uno de sus principales motores. El impacto de grandes proyectos, tales como las represas, generó la aparición de los primeros estudios antropológicos sobre DIDR durante los años 1950, siendo especialmente importante el estudio sobre la represa Kariba (Tonga) de Elizabeth Colson y Thayer Scudder (Colson, 1971). Luego de hacer un seguimiento a cinco comunidades afectadas, concluyeron que el proyecto empobreció a prácticamente toda la población que absorbió los 
costos sociales y que pudo recuperar su bienestar solo después de un largo proceso de adaptación. Estos hallazgos definieron muchos de los estudios posteriores que dan fe del inmenso impacto del desplazamiento y el limitado alcance de los esquemas de reasentamiento (Terminski, 2013).

Debido a ello, principalmente desde el Banco Mundial, se ha buscado incidir sobre una política más comprensiva de DIDR, a partir de los aportes de Michael Cernea, quien sostiene que sus principales efectos han sido el empobrecimiento y el desempoderamiento. Para Cernea, el DIDR no solo debe basarse en el mero reconocimiento de los bienes perdidos, sino en el restablecimiento de su capacidad productiva mediante la reconstrucción de la estructura de oportunidades. Desde la investigación, el modelo propuesto por Cernea ahonda en temas de vulnerabilidad socioeconómica, tales como las pérdidas de sustento económico; el limitado acceso a servicios públicos y mercados; la ruptura de los tejidos sociales; entre otros (Cernea, 1993 y 1999; De Wet, 2006).

\section{EL DIDR EN ENTORNOS URBANOS}

A diferencia del DIDR rural, el DIDR en ciudades fue sistemáticamente subestudiado (Cernea, 1993; Terminski, 2013), ya que se asumía que no afectaba demasiado la economía de los afectados y que era relativamente fácil generar nuevos medios de subsistencia dado el dinamismo de los mercados urbanos (Mejía, 1999; Roquet, Bornholdt, Sirker, y Lukic, 2017). Sin embargo, la especificidad urbana aporta nuevas variables, tales como la poca disponibilidad del suelo en áreas centrales; la relación con las economías informales; los problemas de transporte e infraestructura; el costo de los servicios públicos; la complejidad de las soluciones habitacionales; la heterogeneidad de la población; entre otras. A continuación, exploraremos algunas de ellas.

\section{CENTRALIDAD}

Las poblaciones urbanas tienen formas complejas de sustento, muchas veces basadas en actividades informales que a pesar de su aparente flexibilidad, las hacen depender de la demanda en ciertas zonas céntricas de la ciudad para poder subsistir (Mejía, 1999). No obstante, una práctica común del DIDR ha sido la reubicación en zonas periféricas (Cernea, 1993; Roquet et al., 2017)3. En ese sentido, los efectos de empobrecimiento y marginalización socioeconómica derivados de la distancia -relacionados, por ejemplo, al aumento de los costos y tiempos de transporte dada la lejanía de las fuentes de empleo, la poca demanda laboral o la limitada

3 Suele ser común a nivel mundial encontrar distancias de $10 \mathrm{~km}$ entre los nuevos y antiguos barrios (Cernea, 1993). 
base de clientes en áreas de reubicación- han sido uno de los aspectos más estudiados para los casos de DIDR urbano (Cernea, 1993, 1999; Day, 2013; Abebe y Hesselberg, 2015; Mejía, 1999). Por otro lado, los estudios que se concentran en reubicaciones en áreas centrales han recibido poca atención (Santiago, 1977), probablemente porque se asume que las menores distancias entre las zonas de afectación y reasentamiento mantendrían la relación con mercados y redes preexistentes (Cernea, 1993).

Estos estudios resaltan la necesidad de que la reubicación se dé lo más cerca posible de los asentamientos originales, de modo que se garanticen las oportunidades de los afectados para generar ingresos. También se afirma que muchas de las dificultades de las políticas de DIDR son la falta de suelo disponible en zonas centrales y su elevado valor (Mejía, 1999; Santiago, 1977). Por tal motivo, y tomando en cuenta los altos costos socioeconómicos y culturales que implica la reubicación a zonas cada vez más lejanas, aparece como una alternativa la dotación de vivienda en altura (Mejía, 1999). Sin embargo, esto suele ir en contra de las preferencias de los afectados (Santiago, 1977), deteriorando las relaciones de comunidad y agravando el hacinamiento habitacional (Akça, Fujikura, y Sabbağ, 2012; Abebe y Hesselberg, 2013).

\section{LAS POLÍTICAS DE REUBICACIÓN Y LA VIVIENDA}

A pesar de que el mejoramiento de las condiciones de vivienda es visto por la literatura como uno de los componentes menos complicados del DIDR, este sigue aun siendo poco efectivo (Cernea, 1993); lo cual denota una falta de atención al rol de la vivienda en la recuperación de los afectados. En lugar de generar proyectos con objetivos socioeconómicos, el DIDR urbano suele asumirse solo como la dotación de soluciones que siguen los procedimientos y estándares de la vivienda pública (Koenig, 2002; Mejía, 1999), los cuales simplifican la heterogeneidad y particularidades de las familias sin considerar aspectos tales como las características de sus viviendas anteriores, su composición familiar o sus preferencias (Sepúlveda, 1986; Mejía-Escalante, 2012; Abebe y Hesselberg, 2015).

\section{LA VIVIENDA COMO BASE DEL SUSTENTO FAMILIAR}

La vivienda abarca más aspectos que la habitación, especialmente para los pobres urbanos que cuentan con ella como el activo fundamental para garantizar su sustento económico (Moser, 1998). Este sustento (livelihood) está conformado por 
las iniciativas de subsistencia adoptadas por los hogares

"a fin de asegurar mancomunadamente el logro de uno o varios de los siguientes objetivos: su reproducción biológica; la preservación de su vida; el cumplimiento de todas aquellas prácticas, económicas y no económicas, indispensables para la optimización de sus condiciones materiales y no materiales de existencia" (Torrado, 1982, p. 59).

Por ejemplo, mediante la incorporación de nuevos miembros o la diversificación de sus actividades productivas ante posibles situaciones de estrés económico y social (Moser, 1998; Rakodi, 1999).

\section{POLIITICAS DE COMPENSACIÓN}

Para reducir estos efectos se necesita incorporar nuevos indicadores de evaluación, tales como el valor de los activos familiares y las actividades realizadas en la vivienda (Mejía, 1999). Ello implica la incorporación de los aspectos no-económicos involucrados en las distintas formas del capital (Cernea, 1999), los cuales son "notoriamente complejos, ambiguos y dinámicos" (De Wet, 2006, p. 5). No obstante, los esquemas de compensación se entienden de forma limitada y solo permiten que las personas continúen subsistiendo, en lugar de restablecer o mejorar sus condiciones socioeconómicas previas (Terminski, 2013). A propósito de un estudio sobre el Puente Jamuna en Bangladesh, Al Atahar (2014) encontró que la poca compensación fue una limitante fundamental para la reconstrucción de la vida de los afectados, y que no pudo, por sí sola, evitar el empobrecimiento.

\section{Metodología}

\section{ASPECTOS METODOLÓGICOS PRESENTES EN LA LITERATURA}

Dado el enfoque antropológico presente desde los estudios seminales sobre DIDR, el método más frecuente encontrado en la literatura es el del estudio de caso único de tipo cualitativo (Colson, 1971; Akça et al., 2012; Devitt y Hitchcock, 2010; Abebe y Hesselberg, 2013). Pueden encontrarse también estudios de caso cuantitativos (Day, 2013); cualitativocuantitativos (Amirthalingam y Lakshman, 2009); y estudios comparativos entre la situación antes y después del reasentamiento (Dash, 2008). Hay también evidencia de estudios longitudinales, que, si bien son ideales ya que proporcionan una línea de base precisa, son menos usuales debido a los grandes recursos que implican (Wilmsen y Van Hulten, 2017).

Entre los enfoques de investigación dominantes en el DIDR podemos encontrar el análisis de las condiciones de vivienda postreasentamiento entendida tanto desde las características de la vivienda como a propósito de la satisfacción residencial (Li y Song, 2009); el reasentamiento en relación a las estrategias 
de sustento familiar (Adam, Owen y Kemp, 2015; Amirthalingam y Lakshman, 2009); el aumento de la desigualdad entre los afectados (Akça et al., 2012); los niveles de participación de los afectados (Devitt y Hitchcock, 2010); entre otros.

\section{MÉTODOS EMPLEADOS}

El levantamiento de información se valió de métodos cuantitativos y cualitativos. Se realizaron 201 encuestas estructuradas a 69 hombres y 132 mujeres habitantes de departamentos elegidos aleatoriamente entre junio y septiembre de 2017, todos jefes de hogar. La muestra cubrió el 48.2\% del universo de viviendas de PU, permitiendo un intervalo de confianza de 95\% y un margen de error de 5\%. La encuesta recogió data actual y data retrospectiva (antes del reasentamiento), concentrándose en aspectos físicos de las viviendas tales como sus dimensiones, nivel de consolidación y características espaciales (cantidad de dormitorios y baños); composición del hogar (número de habitantes, estructura familiar); aspectos socioeconómicos y laborales dentro y fuera de la vivienda (ubicación en relación a las fuentes de trabajo y estudio, actividades productivas, percepciones sobre su situación económica); los términos de la compensación recibida y opiniones sobre el proceso de reasentamiento; composición del tejido social (relación con sus vecinos); así como tenencia y propiedad de las viviendas. El conjunto de estos datos configuran el componente descriptivo del estudio.

El análisis se apoyó en un estudio de tipo cualitativo, a través de entrevistas semiestructuradas, que permitieron profundizar en los hallazgos cuantitativos y, a la vez, sirvieron para que posibles casos extremos no sean asumidos como típicos (Maxwell, 2016). La muestra no fue probabilística y se tomó a partir de los participantes de la etapa cuantitativa, eligiendo a quienes ayuden a explicar la data descriptiva ${ }^{4}$. Se alcanzó el punto de saturación teórica luego de 35 entrevistas. De manera complementaria, se acudió a otras fuentes tales como el Censo Nacional 2007 y reportes proporcionados por LAMSAC. Adicionalmente, se realizaron entrevistas con una ex trabajadora y dos representantes de LAMSAC quienes participaron del proceso, y con el exfuncionario municipal a cargo del proceso.

\section{EL HOGAR Y LA VIVIENDA COMO UNIDADES DE ANÁLISIS}

Este estudio toma al hogar y la vivienda como principales unidades de análisis, las cuales han recibido poca atención a diferencia de la comunidad

4 Se utilizaron seudónimos para salvaguardar la identidad de los entrevistados. 
que ha sido la unidad de análisis predilecta dado el fuerte sesgo rural del DIDR. A pesar de ello, ámbitos como el hogar son centrales para medir las consecuencias del desplazamiento, ya que abordan una condición intermedia entre los individuos y la estructura socioeconómica en la que se desenvuelven (Terminski, 2013; Adam et al., 2015). De acuerdo con Adam et al. (2015, p. 3) "los hogares son conceptualizados como unidades complejas que toman decisiones sobre la provisión y cuyo objetivo funcional es dar soporte a la producción y reproducción social". Lo urbano impone un importante grado de autosuficiencia económica a nivel del hogar como unidad productiva y reproductiva (Vaughan, 1983).

\section{LIMITACIONES DEL ESTUDIO}

Este no es un estudio longitudinal, y el total de la data retrospectiva está basada en el recuerdo de los encuestados, lo cual puede contener sesgos de memoria y subjetividad (Wilmsen y Van Hulten, 2017). Por tanto, el componente retrospectivo no considera variables relacionadas a opiniones emocionales tales como el grado de satisfacción en sus antiguos barrios y viviendas (Blane, 1996). La falta de estudios previos y la imposibilidad de acceder a encuestas de línea de base no permitieron cruzar los hallazgos de la encuesta con información anterior. Por tanto, y a pesar de que fueron recogidos a partir de recuerdos relativamente recientes (de tres a cuatro años), todos los datos anteriores a la reubicación son aproximados.

Una versión preliminar de la encuesta fue aplicada a una muestra de 15 familias, lo cual evidenció la dificultad de recoger datos relacionados a la valorización de las viviendas, así como los niveles de ingreso debido a los problemas de memoria mencionados y a la sensación de incomodidad que se generó. Una variable frecuente en la literatura que tampoco fue tomada en cuenta es la distancia, ya que se asume que no hubo un considerable desplazamiento, lo cual fue luego corroborado con los hallazgos. Debido a que, según la literatura, la ubicación de los proyectos de reasentamiento en zonas centrales tiende a correlacionarse con temas de vivienda, se prestó más atención a dicho componente. Otras variables comunes al DIDR son las relaciones a nivel comunal/barrial. Estas fueron excluidas, ya que consideramos que, dado lo reciente de la reubicación, fue muy pronto para evaluar comparativamente impactos a nivel comunitario, ya que las familias se encontraban aun en proceso de adaptación luego de haber habitado sus barrios de origen durante períodos mayores. 


\section{Caso de estudio: el Proyecto Línea Amarilla y el Complejo Patio Unión}

"Línea Amarilla" es un megaproyecto de infraestructura de transporte que une el este de Lima con el Callao, incluyendo la zona del centro histórico, a la que atraviesa mediante un túnel de casi dos kilómetros por debajo del río Rímac. El proyecto se estructuró como Asociación Público Privada (APP) y se adjudicó a la multinacional brasileña OAS mediante la conformación de su subsidiaria LAMSAC, que reubicó 1.348 viviendas en diferentes zonas de la ciudad entre 2013 y 2015.

La zona de afectación es una franja de aproximadamente 4.5 kilómetros ubicada junto al río Rímac y a muy poca distancia del Centro Histórico. Está conformada por aproximadamente 25 asentamientos de distintas características en relación a su grado de consolidación; su dotación de servicios básicos; su reconocimiento legal; su condición de riesgo físico y sus niveles socioeconómicos y de desarrollo humano (Strauch, Takano y Hordijk, 2015). Debido a una situación previa de inviabilidad social, se optó porque sea LAMSAC la que ejecute el proceso en lugar de la Municipalidad (Takano, 2017). Al tratarse de una APP autofinanciada, LAMSAC tuvo la potestad de implementar su propia política de reasentamiento, no estando requerida de ejecutar un Plan de Compensación y Reasentamiento Involuntario (PACRI), obligatorio por ley para proyectos financiados con fondos públicos y alineado con buenas prácticas internacionales. El proceso se basó en un esquema de compensación que tomó como unidad básica el predio con propiedad registrada mediante la figura de un propietario/un compensado, tal como indica el marco legal peruano (Ley 27.628).

Del número total de predios reubicados, 827 fueron compensados en efectivo, 36 fueron expropiados, 378 fueron reubicados en Patio Unión (PU), 47 en otro complejo llamado Acomayo, y 60 en otras zonas de la ciudad según las preferencias y posibilidades de las familias. El universo del estudio corresponde a los reubicados en PU, ubicado muy cerca de la zona de afectación (Figura 1), y que alberga al 28\% del total de predios afectados: 378 reubicaciones en 417 departamentos valorizados en USD $30.000^{5}$. Este consta de 18 edificios de seis plantas, agrupados en tres etapas donde se localizan 81, 168 y 168 departamentos respectivamente. Los departamentos tienen un área de $60 \mathrm{~m} 2$ sin posibilidad de ampliación y están compuestos por

5

El número de departamentos excede a las familias de origen, ya que una cantidad de familias recibió más de un departamento como compensación por sus predios. 


\section{FIGURA 1. UBICACIÓN DEL ÁREA DE AFECTACIÓN Y DE PATIO UNIÓN.}

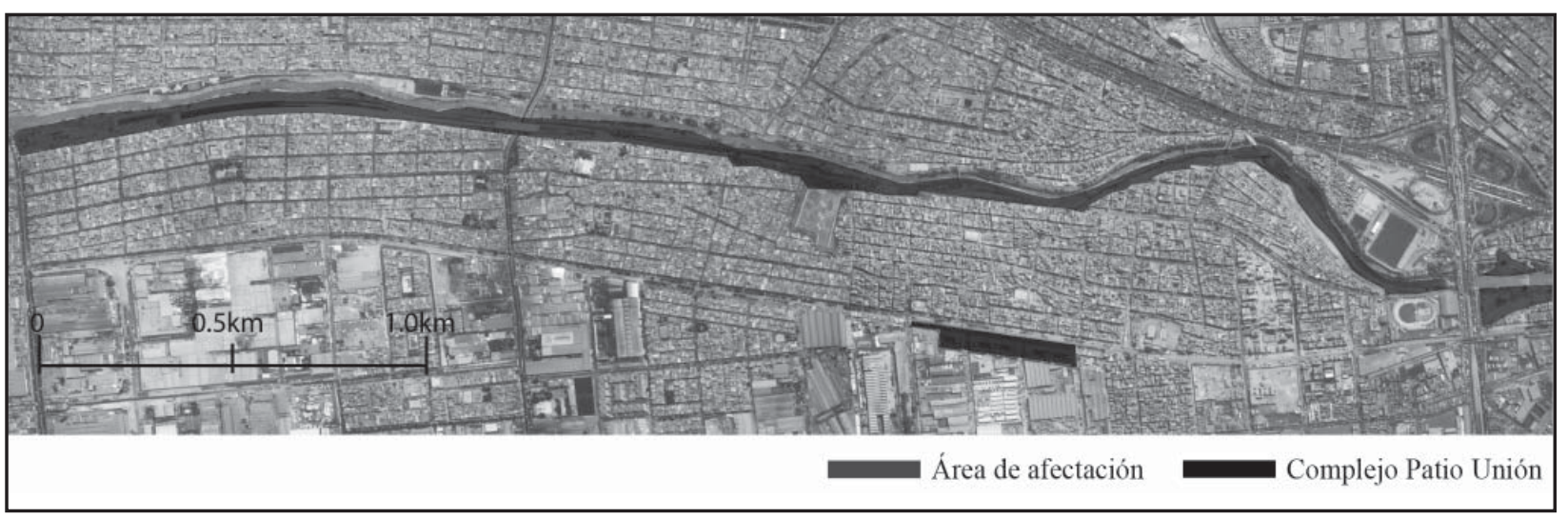

Fuente: Elaboración propia en base a Bing Maps Aerial

una sala con comedor, una cocina, un baño, dos dormitorios y un estudio pequeño.

A continuación se presentarán los principales hallazgos del estudio, los cuales exploran las variaciones en la habitabilidad de las viviendas, relacionadas a la estructura familiar y a aspectos económicos y productivos. Luego se presentarán las percepciones sobre el proceso de reasentamiento para, finalmente, analizar los impactos socioespaciales de dicho proceso.

\section{VARIACIONES DE HABITABILIDAD}

En relación a las variaciones en términos espaciales y funcionales antes y después del reasentamiento, las viviendas existentes tenían en promedio más del doble del área de los departamentos actuales; más de 50\% de baños y más de un dormitorio extra (tabla 1). A pesar de que el número promedio de habitantes de estas era casi 50\% mayor, el área promedio por habitante disminuyó en $29.6 \%$ luego de la reubicación hasta llegar a $14.12 \mathrm{~m} 2$ en una ciudad cuyo promedio es de $27 \mathrm{~m} 2$ ("Crecimiento urbano y acceso a oportunidades", 2017). Actualmente, 38\% de los departamentos de Patio Unión tienen cinco o más habitantes.

Las percepciones sobre la cantidad de espacio disponible en los departamentos siguen la misma dirección que los datos de la Tabla 1. Mientras 71.5\% de los encuestados opina que les falta espacio a 
diferencia de sus viviendas anteriores, hubo una reducción de $\mathrm{m} 2 /$ hab en $74.6 \%$ de los casos. Eliana, quien antes habitaba una casa consolidada de $180 \mathrm{~m} 2$, refleja dicho sentir sobre el departamento que recibió:

"Muy chiquito es. Mis nietecitos van creciendo [...] somos seis: tres niños y tres adultos. Son cuartos chiquitos. Estamos en camarotes con una mesita al costado [...] En mi casa cada uno tenía su cuarto y su cama. Ahora compartimos, yo con mi nieta en un cuarto, mis nietos solitos en otro cuarto, y mi hija con su esposo en el tercero".

A pesar de las menores dotaciones de $\mathrm{m} 2 / \mathrm{hab}$, el promedio de habitantes por dormitorio bajó de 1.62 a 1.54 habitantes. Ello podría deberse a que las antiguas viviendas tenían dormitorios más grandes y más áreas comunes, lo cual es fundamental considerando la gran cantidad de familias extendidas que las habitaban. En la zona de afectación vivían 1.38 hogares por cada vivienda y al menos 24\% de la población residente no era parte de las familias nucleares principales (Walsh Peru S.A., 2011; Instituto Nacional de Estadística e Informática [INEI], 2007).

\section{LA EXCLUSIÓN DE LA FAMILIA EXTENDIDA}

Solo 854 de 1.274 miembros de los hogares encuestados pudieron ser reubicados en PU, mientras los 421 restantes tuvieron forzosamente que buscar vivienda en otro lugar. Esto hizo que el número de habitantes del predio promedio se redujera en 2.1 personas (33\%) luego del traslado. El testimonio de Amelia, quien vivía en una casa de 130 metros cuadrados (dos pisos), evidencia el desmembramiento de su familia debido a la falta de espacio:

\footnotetext{
"Actualmente somos cinco personas viviendo en el departamento. Antes vivíamos ocho personas pero en una casa de 200 metros cuadrados. Arriba tenía como cinco dormitorios; allí estaba mi hijo con sus hijitas [...]. Ahora tuvimos que separarnos porque no hay espacio. Mi hijo paga 300 soles ( USD90) en la casa de un familiar de su esposa. Están en un cuartito que está dividido; no tiene cocina, comen en la calle y lo usan solamente para dormir".
}

De las personas que no lograron reubicarse en PU, actualmente $48.8 \%$ alquila una vivienda; $20 \%$ se mudó con un familiar; 20\% compró una nueva propiedad o invadió un terreno y 11.2\% falleció. Considerando que solo $11.9 \%$ de los propietarios recibió algún tipo de compensación aparte de los departamentos, se asume que gran parte de esta población excedente no fue compensada.

\section{LA VIVIENDA Y EL SUSTENTO FAMILIAR}

Setenta encuestados tenían negocios en sus viviendas antiguas (34.8\% del total), de los cuales 51 eran tiendas, 13 eran talleres y seis subarrendaban habitaciones. 47 de ellos tuvieron que cerrar luego del reasentamiento y en 23 casos, las familias aun conservan sus negocios (17 en Patio Unión y 


\section{TABLA 1. VARIACIONES DE LOS INDICADORES DE HABITABILIDAD DE LAS VIVIENDAS ANTES Y DESPUÉS DE LA REUBICACIÓN ( $\mathbf{N}=201)$}

\begin{tabular}{|l|lll}
\hline & $\begin{array}{l}\text { Antes de la } \\
\text { reubicación }\end{array}$ & $\begin{array}{l}\text { Después de la } \\
\text { reubicación }\end{array}$ & Variación porcentual \\
\hline Total de habitantes & 1.274 personas & 854 personas & $-33 \%$ \\
\hline Promedio de número de habitantes por vivienda & 6.34 personas & 4.25 personas & $-33 \%$ \\
\hline Promedio de área de vivienda & $127.22 \mathrm{~m} 2$ & $60.00 \mathrm{~m} 2$ & $-52.8 \%$ \\
\hline Promedio de área por habitante & $20.06 \mathrm{~m} 2$ & $14.12 \mathrm{~m} 2$ & $-29.6 \%$ \\
\hline Promedio de número de dormitorios & 3.9 dormitorios & 2.76 dormitorios & $-29.2 \%$ \\
\hline Promedio de número de baños & 1.54 baños & 1 baño & $-35.0 \%$ \\
\hline Promedio de habitantes por dormitorio & 1.62 personas & 1.54 personas & $-4.9 \%$ \\
\hline Promedio de habitantes por baño & 4.11 personas & 4.25 personas & $+3.4 \%$
\end{tabular}

Fuente: Elaboración propia 
seis en otras zonas). Aproximadamente cerraron 1.2 tiendas por cada tienda que se trasladó a Patio Unión. Para el caso de los talleres, esta relación fue de tres a uno y para el caso de las familias que alquilaban habitaciones, dicha fuente de ingreso se perdió completamente. El testimonio de Tamara da una idea del impacto de la pérdida de sus negocios:

"Tenía un negocio de venta en la puerta de mi casa. Primero era tipo tienda y después tipo restaurante [...]. Vivíamos de eso. Ahora mis hermanas trabajan cada una por su cuenta pero nos da menos. Ya no se puede pagar la universidad; sale solo para los gastos de la casa".

Entre las razones de cierre de negocios luego de la reubicación, las familias mencionan la poca demanda en PU, la falta de espacio o problemas de compatibilidad de usos semiindustriales con los edificios multifamiliares, tomando en cuenta que no se implementó ningún espacio especializado para ello. Según los entrevistados, el valor intrínseco de los negocios y el lucro cesante derivado de su pérdida no fueron reconocidos, sino únicamente las funciones de vivienda, lo que generó un descontento generalizado, tal como comenta Amelia, quien solo recibió un departamento en compensación:

"Mi negocio lo he suspendido; ya no puedo trabajar aquí. Fabricaba muñecas y peluches. A pesar de que ellos vieron todas mis máquinas en mi antigua casa, no me dieron espacio [...] ¿Dónde pongo la máquina? ¿Dónde pongo ahora la mesa para trabajar?".
Mientras 45.6\% de las familias que realizaban actividades económicas en sus viviendas siente que su situación ha empeorado, los impactos económicos en aquellas familias que no tenían viviendas productivas parece ser menor, ya que solo $20.2 \%$ cree que su situación es peor que antes. Haber permanecido en el centro de la ciudad es un factor importante en la satisfacción de este último grupo, considerando que 48.6\% de los encuestados trabaja en el Cercado de Lima. Ello les permitió conservar sus redes económicas y patrones de movilidad, tal como manifiesta Emma, cuyo esposo trabaja en el centro de la ciudad:

"Hubiésemos preferido un terreno, pero nos querían mandar por Ventanilla [periferia norte de la ciudad]. Entonces imagínate, ¿quién va a querer vivir tan lejos? Por eso, así nos lamentemos, escogimos el departamento por estar cerca al trabajo de mi esposo y el colegio de mis hijos".

\section{LAS PREFERENCIAS FAMILIARES Y LA COMPENSACIÓN}

Del total de encuestados, $52.5 \%$ opina que la compensación otorgada fue injusta y solo $14.7 \%$ estuvo satisfecho con la opción del departamento, mientras $52.8 \%$ hubiera preferido una casa y $25 \%$ un terreno para construir. Entre las razones por las cuales eligieron el departamento, 37\% consideró como primera opción el hecho que la compensación era insuficiente y ello no les permitió optar por algo de acuerdo a sus preferencias; $28.6 \%$ consideró que los terrenos disponibles estaban demasiado lejos; 
18.2\% considero que el departamento era la única opción disponible y 16.2\% mencionó otra razones. El testimonio de Emma evidencia que la ubicación céntrica de Patio Unión fue un factor importante a la hora de optar por el departamento, tomando en cuenta que la distancia promedio entre el complejo y sus antiguas viviendas era de solo 850 metros.

A las familias aptas para recibir un departamento se les dio la opción de comprar otra propiedad de USD 30.000. Sin embargo, el valor del suelo construido en áreas cercanas era de USD900 por $\mathrm{m} 2$. Eso significa que para la familia promedio, el reubicarse en una vivienda equivalente, hubiera tenido un costo de USD114.000, lo cual estaba fuera de los términos de la negociación. Tal como manifiesta Ada, quien tenía una vivienda consolidada de 200 metros cuadrados donde vivían ocho personas y recibió solo un departamento a cambio:

"La cuestión era económica; no alcanzaba porque nos daban solo USD30.000. Por eso nos pusimos de acuerdo con mi hermana y dijimos: mejor agarramos el departamento y después cuando nos den el título lo vendemos. ¿Por qué? Porque necesitamos más espacio; ojalá que se pueda. Si no, ya nos quedaremos con esto".

"A mí me gustaría mudarme porque en mi familia somos siete y si tuviera una casa, tendría los aires. La casa que tenía era de dos pisos pero tenía los aires para seguir construyendo para mis hijos. Acá en un departamento no se puede." (María).
"En esa construcción hemos invertido pues; los hijos trabajando hemos construido eso. Entonces es un patrimonio para la herencia de los hijos. La empresa no nos quiso reconocer y nos dijo: que los hijos se busquen su casa. Y yo le dije: ¿cómo? ¿Tú nos vas a despojar de la herencia que nos corresponde de nuestros padres? ¡No! te equivocas. [...] Era una pelea con la empresa" (Eladia, quien recibió un departamento a cambio de una vivienda de $120 \mathrm{~m} 2$ y dos pisos).

El esquema de compensación solo consideró a la vivienda como un activo físico presente mas no como un valor futuro. En ese sentido, la declaración de María y Eladia evidencian su importancia como un legado para sus descendientes, de modo que puedan iniciar su vida familiar a menor costo.

\section{ESTRATEGIAS DIFERENCIADAS DE NEGOCIACIÓN}

Dadas las diferencias entre la población afectada, queda claro que no todos los predios fueron compensados de la misma manera sino que algunos recibieron más, no solo como reflejo del valor de sus viviendas sino también de estrategias de negociación diferenciadas (Strauch et al., 2015). En consecuencia, mientras la mayoría de afectados recibió un departamento en compensación (92.9\%), otros recibieron dos (5.3\%), tres (1.3\%), cuatro $(0.25 \%)$ y siete departamentos (0.25\%). 
"El problema era que había una directiva que no trabajaba, solamente se dedicaba a pedir plata. Entonces cada uno se apartó, cada uno hacía sus cosas independientemente. Porque si hubiera sido conjuntamente, la negociación hubiera sido diferente" (Amelia, quien recibió un departamento a cambio de una vivienda de $120 \mathrm{~m} 2$ ).

"Tuvimos que negociar para recibir cuatro departamentos. Al principio nos quisieron dar uno, después dos. Mis hermanas eran las que negociaron a cuatro. Incluso ellos (LAMSAC) mismos querían dividir a los hermanos." (Marina, quien recibió cuatro departamentos a cambio de una vivienda de $130 \mathrm{~m} 2$ ).

"Los primeros que se fueron de allá fueron mal pagados; tremendas casotas que tenían. Vivían dos, tres familias y solo les reconocieron un departamento" (Tamara, tercera etapa, cuya familia recibió tres departamentos por una vivienda de $160 \mathrm{~m} 2$ ).

Según varios entrevistados, el punto de partida estratégico de LAMSAC fue negociar individualmente (trato directo) ofreciendo un departamento por cada propietario sin considerar la cantidad de personas/familias ocupantes o la estimación del valor de las propiedades. Para una informante de LAMSAC, la negociación individual era inevitable ya que la relación con las organizaciones barriales se había roto luego de un conflicto social y no veían otra manera de retomar el proceso. Por otro lado, debido a la falta de información disponible, la población no tomó iniciativas organizadas porque no se sabía exactamente quien sería afectado y quién no (M. Silva, comunicación personal, 30 de noviembre de 2017; ver también Strauch et al., 2015). La atomización y secretismo de la negociación, generaron desconfianza, no solo hacia la empresa, la municipalidad o los dirigentes sino también entre los mismos vecinos, lo cual ha tenido efectos socioespaciales en Patio Unión.

\section{EFECTOS SOCIOESPACIALES}

Aquellas familias que recibieron mayores compensaciones fueron reubicadas en la segunda y tercera etapa. $27 \%$ de los departamentos que fueron dados a más de un propietario se encuentran en la segunda etapa, $73 \%$ en la tercera y ninguno en la primera. Según Waldo, quien recibió un departamento a cambio de una vivienda consolidada de $100 \mathrm{~m} 2$ :

"A mí me dieron en compensación este departamento, pero había otra vecina de un costado que no tenía ni siquiera la mitad; su casa era de adobe mientras la mía era de ladrillo. A mí me dieron este departamento valorizado en USD 30.000 y a esa vecina le dieron USD 57.000 en efectivo. Me he enterado que a otros les ha pasado; les han dado hasta dos, tres departamentos. Han negociado y en esa negociación, los que han venido últimos han ganado y los que hemos venido primero hemos perdido".

Sin embargo, la evidencia arroja que aquellos que se mudaron después tenían viviendas más grandes y familias más numerosas. Por ejemplo, las viviendas de los que hoy habitan la tercera etapa eran 
21.6\% más grandes y tenían 31.3\% más habitantes que los de la primera. Por tanto, el hecho que hayan esperado más tiempo para negociar puede estar relacionado con la defensa de sus legítimos intereses más que con un comportamiento oportunista, ya que estas familias tenían más que perder.

Las percepciones sobre la inequidad de las compensaciones han fortalecido dinámicas preexistentes de estigmatización hacia barrios considerados como socialmente problemáticos debido a que la estrategia implementada por LAMSAC implicó que estos barrios sean dejados para el final y, en consecuencia, reubicados también en la tercera etapa. Entre ellos, puede mencionarse el barrio Agnoli, donde los habitantes en 2007 tenían una tasa de desempleo $72.4 \%$ mayor que el promedio del área de afectación (INEI, 2007). Hay 12 familias encuestadas provenientes de este barrio, de las cuales tres viven en la segunda etapa y nueve en la tercera.

"En parte de la etapa dos y la etapa tres hay gente de Huascarán, y lo peor es que han traído gente de Agnoli, que es la zona más difícil quizá de Lima [...] Incluso se habla de que han sacado gente que ha estado vendiendo droga. Se habla que en el tercer lote, en el quinto piso hay un prostíbulo" (José, primera etapa).

"Aquí [en la primera etapa] son amistosos. En el primer block sí, pero de atrás para allá... ¡wow! [...] En la tercera etapa, si vas para allá vas a ver que hay un basural en la cochera. Roban, todo se llevan de acá.
Por eso hemos puesto rejas y las mantenemos con llave" (Catalina, primera etapa).

Y por otro lado... "los de la primera etapa se han independizado, y casualmente les dicen hasta los 'gringos' porque nadie entra. Incluso ellos mismos manejan su llave y quieren hacer su propio condominio interno. Al hacer eso, los otros quieren hacer lo mismo. No hay unión y así no vamos a llegar a ningún lado" (Gloria, tercera etapa).

\section{LOS SERVICIOS COMUNES Y LA RELACIÓN CON LOS VECINOS}

Además de los problemas de estigmatización antes mencionados, la gestión de responsabilidades colectivas tales como el pago de servicios, impactan en el sentido de comunidad. Por ejemplo, ya que las familias no cuentan aún con la propiedad de sus departamentos y los recibos de agua potable llegan a nombre de LAMSAC, estas tienen que organizarse para dividir el pago en partes iguales. Ello genera una sensación de descontento ya que muchas familias sienten que están subsidiando a otras que consumen más, tal como evidencia el testimonio de Julia:

"Yo acá tengo mis dos hijitos no más, pero pago igual como si vivieran dos familias. Ellos son como 20 y aquí hay cuatro. Los 20 se bañan, se duchan y yo les estoy pagando el agua".

$54.5 \%$ de familias piensa mudarse apenas reciban un título de propiedad que les permita transferir 
sus departamentos, lo cual refleja un sentido de insatisfacción. A continuación, desarrollaremos las posibles razones de la insatisfacción al interior de PU, así como su relación con el proceso de compensación y reubicación.

\section{Discusión}

Tal como se mencionó en la revisión de literatura, la cuestión urbana aporta una serie de particularidades a los estudios sobre DIDR, las cuales no han recibido suficiente atención debido a un sesgo rural que tiende a generar interpretaciones poco comprensivas. Si bien los procesos debieran apuntar no solo a la restitución de los bienes perdidos o al restablecimiento de las condiciones de vida previas, sino a permitir que las personas reconstruyan y mejoren sus sustentos de vida (Cernea, 1999); el DIDR urbano se ha tendido a concentrar únicamente en la provisión de viviendas, ajustada a la lógica de la vivienda pública masiva de manera inequívoca (Roquet et al., 2017; Mejía, 1999). Esta se concibe como una unidad hecha exclusivamente para habitar y se basa en la concepción de unidades estandarizadas y poco flexibles que no empatan con las preferencias de los hogares (Sepúlveda, 1986).

Ello se hace más evidente, considerando que el DIDR es un proceso de tipo involuntario. Al no poder elegir, o tener sus opciones severamente limitadas, los hogares se ven obligados a adaptarse a estos nuevos espacios. El problema es que la aproximación de dichos hogares a la vivienda suele ser opuesta, en tanto que gira alrededor de la transformación del espacio de acuerdo a sus necesidades (Sepúlveda, 1986), y no al contrario. En ese sentido, la poca comprensión de los múltiples roles que cumple la vivienda -tales como ser el principal activo físico del sustento familiar, el bien que los hijos heredarán o simplemente el lugar donde hacer una fiesta con la familia- genera desencuentros que comprometen no solo la calidad de vida sino las estrategias de subsistencia de los afectados, lo que representa severos riesgos de empobrecimiento (Cernea, 2000). Por ello, consideramos esencial hacer una revisión de las limitaciones de esta aproximación y el modo cómo han afectado a las personas. Los aportes de la literatura sobre vivienda al DIDR urbano son importantes para entender una serie de relaciones que suelen pasar desapercibidas, tales como los efectos espaciales de la reubicación dentro de la vivienda y su relación con las dinámicas socioeconómicas de los hogares que las habitan.

En primer lugar, la elección del hogar como unidad de análisis permitió capturar con precisión la dinámica de la relación entre las personas y el espacio doméstico, en el sentido que fue una escala idónea para registrar y comparar la evolución de las variables. Esto también permitió hacer una observación transversal a todos los hogares de la muestra, evidenciando su heterogeneidad. 


\section{LAS PREFERENCIAS DE LOS HOGARES Y LA COMPENSACIÓN}

En general, los hogares urbanos presentan una serie de particularidades en relación al número de habitantes y composición familiar (Driant y Riofrío, 1987); sus estrategias de sustento y activos familiares (Moser, 1998; Rakodi, 1999); su concepción de la vivienda y la familia como proyecto de alcance intergeneracional (Wheelock y Oughton, 1996) o sus capacidades de negociación (Koenig, 2002). Para el caso estudiado, estas diferencias contribuyeron a definir preferencias específicas de reasentamiento, configuradas a partir de la ubicación céntrica de las alternativas disponibles; sus dimensiones y posibilidades de crecimiento; o su capacidad para ser utilizadas como medio de producción e inversión, aportando al bienestar futuro de la familia.

El espíritu del programa de reasentamiento implicó que los afectados elijan "la mejor alternativa para sus necesidades" luego de contrapesar dichos factores ("Programa de reubicación de viviendas", 2017), ya que, mientras mayores sean las alternativas de reasentamiento, las posibilidades de aminorar los impactos negativos serán mayores dada la heterogeneidad social (Mejía, 1999). El problema es que en muchos casos, estas opciones estuvieron principalmente limitadas por los montos de compensación. LAMSAC tuvo un importante incentivo para que las compensaciones sean en principio las menores posibles, ya que los costos de reasentamiento no fueron incorporados en la estructuración financiera del megaproyecto LA, lo cual significó que mayores compensaciones habrían afectado la tasa de retorno de la concesión.

Ello se canalizó mediante tres estrategias: en primer lugar, en 2007 se revocó la zonificación residencial del área de afectación en función de reducir el valor de las viviendas (Strauch et al., 2015); segundo, la capacidad de elección de los afectados se vio limitada ya que el predio donde está Patio Unión fue donado por la Municipalidad de Lima a LAMSAC, lo cual implica que la opción de los departamentos contenía un subsidio oculto y alternativas cercanas por USD30.000 eran inviables. Finalmente, la atomización de la negociación minimizó los rendimientos crecientes a escala de una negociación colectiva, al dejar que cada familia mejore su posición de manera independiente. Adicionalmente, los bajos niveles de representación y el comportamiento oportunista de los líderes locales afectaron el tejido social y facilitaron las estrategias de 'divide y conquistarás' por parte de actores como LAMSAC (Koenig, 2002, p. 9).

La percepción generalizada de injusticia a propósito de las compensaciones puede explicarse desde el diseño del esquema de compensación que puso el valor de mercado (de intercambio) de los activos por delante del valor social (de uso) reconocido por las familias, el cual tendió a ser mucho mayor (Al 
Atahar, 2014; Terminski, 2013). A diferencia, de los métodos usuales de análisis costo-beneficio que no son capaces de capturar aspectos tales como la pérdida o el costo de recuperación de los afectados, la construcción de dicho valor tiene dimensiones tanto económicas como no económicas (Cernea, 1999).

La dimensión económica tuvo un importante rol sobre la decisión de las familias, que estuvo fuertemente influenciada por las ventajas de centralidad de Patio Unión. Si bien las economías informales pueden permitir un relativo nivel de movilidad, tienden también a generar dependencia territorial, y en ese sentido la pérdida de fuentes de ingreso y empleo suele ser vista como un factor más relevante que la pérdida misma de vivienda en una ciudad donde el $60 \%$ de la población pertenece al sector informal (Cernea, 1993; INEI, 2014;).

El reconocimiento de la centralidad de los barrios debiera ser el punto inicial de cualquier proceso de reasentamiento (Abebe y Hesselberg, 2013), tomando en cuenta los altos riesgos socioculturales derivados del desplazamiento a zonas alejadas (Cernea, 1999; Mejía, 1999). No obstante, el caso estudiado evidencia que la sola implementación de un proyecto de reasentamiento en una zona central no garantiza dicho desarrollo. Por el contrario, las restricciones derivadas del uso intensivo del suelo podrían limitar el alcance de las soluciones (Koenig, 2002).

\section{SOBRE LOS EFECTOS DEL REASENTAMIENTO}

El beneficio para los hogares, derivado de la centralidad se dio a cambio de sacrificar la habitabilidad de sus nuevas viviendas, aumentando la densidad y obligando a las familias a desmembrarse. Considerando un escenario, en el que ningún afectado haya tenido que salir de PU, el índice de ocupación hubiera pasado de 4.25 a 6.34 personas por departamento, y el área por habitante, de $14.12 \mathrm{~m} 2 \mathrm{a}$ $9.46 \mathrm{~m} 2$. Los miembros de los hogares marginados fueron expuestos a una condición socioeconómica vulnerable ya que sus únicas opciones eran ocupar nuevo suelo en cada vez peores condiciones (Ramírez-Corzo y Riofrío, 2006); alquilar habitaciones o viviendas en condiciones de hacinamiento (CNDH, 2002 en Riofrío, 2004); incorporarse en otros hogares como allegados en condiciones desventajosas (Necochea, 1987); o asumir los costos de la compra de una vivienda.

Además, sea por falta de espacio o poca demanda, muchas viviendas dejaron de operar como medios de producción, exponiendo a los hogares a situaciones de vulnerabilidad (Moser, 1998; Rakodi y Lloyd-Jones, 2002; Siegel, 2005). Ello coincide con los hallazgos de Mejía-Escalante (2012) para un caso en Medellín-Colombia, donde evidencia la pérdida de sustentos económicos debido a que la tipología 
de vivienda postreasentamiento no se adaptó a las dinámicas preexistentes, encontrando que a diferencia de las antiguas viviendas que permitían la subdivisión y el crecimiento en altura, sea para albergar a nuevos miembros de la familia o desarrollar actividades económicas; los nuevos apartamentos solo permiten incorporar estas nuevas actividades sin poder separarlas de aquellas existentes, lo cual afectó la calidad de vida de la población.

Las compensaciones diferenciadas generaron un escenario postreubicación conflictivo, el cual se encuentra influenciado por la constante comparación entre los afectados (Jiang, Waley y Gonzalez, 2018). En ese sentido, coincidimos con De Wet (comunicación personal como se cita en Koenig, 2002, p. 23) cuando encuentra que para un caso de estudio en Lesotho, las variaciones en las compensaciones pudieron haber afectado la solidaridad entre los reubicados. A las percepciones negativas relacionadas al supuesto oportunismo de los habitantes de la tercera etapa, se sumaron dinámicas de estigmatización asociada a los barrios de origen de las familias que también, debido al diseño mismo del proceso, fueron reubicados allí. La estigmatización barrial ha cambiado su escala y se ha reproducido dentro de Patio Unión con efectos de microsegregación que impactarían negativamente en la adaptación social y especialmente en la recuperación económica debido a que tienden a socavar la conformación de nuevo capital social (Cernea, 2000), algo que debió planificarse de antemano.

\section{Conclusiones y recomendaciones}

Tomando en consideración los efectos que tuvo la reubicación en las familias afectadas debido a un proceso enfocado en lo estrictamente compensatorio, es necesario que no solo se reconozca el valor de los activos perdidos, sino se diseñe a detalle el proceso de recuperación de las oportunidades perdidas en relación a la ubicación de los barrios, a la vivienda como base del sustento familiar, a la separación forzosa de las familias, a las redes económicas y el empleo, y al desgaste del tejido social. El problema es que en un contexto de altos precios del suelo y especulación urbana, es cada vez más costoso implementar esquemas de reasentamiento adecuados, considerando que los recursos disponibles suelen ser insuficientes y están limitados solo a temas de compensación y expropiación. Por ejemplo, para una muestra de proyectos en Latinoamérica y el Caribe del Banco Mundial, se estimó que la subestimación de los costos era de 54\% en promedio (Mejía, 1999).

En ese aspecto, coincidimos con Cernea (2003, p. 39), cuando afirma que la concepción de los proyectos debe pasar "de una economía basada en la compensación a una basada en el aumento del ingreso, para así lograr un reasentamiento con desarrollo", que reconozca a la vivienda como el 
soporte de un sistema no solo reproductivo sino también productivo. Para ello, es necesario que las dimensiones del DIDR sean precondiciones necesarias para la aprobación de los componentes sociales de los estudios de impacto ambiental, y, más aún, que sus costos se internalicen en la estructuración financiera de los proyectos de infraestructura (Mejía, 1999). Si los costos sociales fuesen demasiado altos, los proyectos ya no serían rentables.

Ello también implica la profundización del acompañamiento social de los procesos mediante protocolos de reasentamiento que se amparen en mecanismos censales claros, que hagan un seguimiento por familia y no por predio catastral. El enfoque de compensación por predio se suele justificar afirmando que agiliza los procesos y establece cierto orden, considerando que el valor de los activos es difícil de reconocer en entornos urbanos y siendo relativamente sencillo mentir sobre la cantidad de ocupantes y bienes para recibir mayores compensaciones (Crisp, Morris y Refstie, 2012; Koenig, 2002). Sin embargo, tal como muestra la evidencia de caso, este enfoque presentó esencialmente dos problemas: no reconoció la complejidad al interior de los hogares y socavó la integración social de los hogares reubicados.

A diferencia del predio como unidad física, la familia requiere de un proceso complejo de visibilización, que necesita de la participación activa de los afectados desde el inicio, potenciando la comunicación y evitando el oportunismo. No obstante, la implementación de mecanismos de participación en DIDR aún se entiende como algo meramente retórico y no negociable (Oliver-Smith, 2006). Eso también es sintomático de los mecanismos de APP, que tienden a subfinanciar los aspectos sociales del desarrollo de infraestructura en general, ya que a diferencia de los proyectos públicos o aquellos financiados por la banca de fomento, estos no requieren ajustarse a los estándares del DIDR, ya que su casi único objetivo es el retorno económico (Strauch et al., 2015; Takano, 2017). La incorporación de regulaciones e instrumentos de gestión social tales como los PACRI, no deben limitarse solo a los proyectos de inversión pública sino extenderse al universo de las APP y proyectos privados.

Se requiere construir una relación orgánica entre las empresas, los gobiernos locales y la población organizada, de modo que se garantice la justicia de las compensaciones y el derecho a las oportunidades económicas en un escenario en el que, dada la escala de los procesos de reestructuración metropolitana y desarrollo de grandes proyectos de infraestructura urbana, los impactos del DIDR tenderán a ser cada vez mayores.

\section{AGRADECIMIENTOS}

El autor agradece la labor de campo realizada por Juan Carlos Corrales y Janet Medina junto con el apoyo económico del Instituto de Investigación Científica (IDIC) de la Universidad de Lima. 


\section{Referencias bibliográficas}

Abebe, G., y Hesselberg, J. (2013). Implications of urban development-induced resettlement on poor households in addis ababa. Ghana Journal of Geography, 5, 32-50.

Abebe, G., y Hesselberg, J. (2015). Community participation and inner-city slum renewal: relocated people's perspectives on slum clearance and resettlement in Addis Ababa. Development in Practice, 25(4), 551-562. http://dx.doi.org/10.1080/096 14524.2015 .1026878 .

Adam, A., Owen, J., y Kemp, D. (2015). Households, livelihoods and mining-induced displacement and resettlement. The Extractive Industries and Society, 2(3), 581-589. https://doi.org/10.1016/j. exis.2015.05.002.

Akça, E., Fujikura, R., y Sabbağ, Ç. (2012). Atatürk dam resettlement process: increased disparity resulting from insufficient financial compensation. International Journal of Water Resources Development, 29(1), 101-108. https://doi.org/10.1080/07900627 .2012 .738497$.

Al Atahar, S. (2014). Development-driven forced displacement and compensation-based resettlement: experiences from the Jamuna multi-purpose bridge project. Development in Practice, 24(2), 258-271. https://doi.org/10.1080/09614524.2014.887660.

Amirthalingam K., y Lakshman R. (2009). Displaced livelihoods in Sri Lanka: An economic analysis. Journal of Refugee Studies, 22(4), 502-524. https:// doi.org/10.1093/jrs/fep031.
Blane, D.B. (1996). Collecting retrospective data: Development of a reliable method and a pilot study of its use. Social Science \& Medicine, 42(5), 751-757. https://doi.org/10.1016/0277-9536(95)00340-1.

Cernea, M.M. (1993). The urban environment and population relocation. Washington, D.C.: The World Bank.

Cernea, M.M. (1999). Why economic analysis is essential to resettlement: A sociologist's view. Economic and Political Weekly, 34(31), 2149-2158. Recuperado de www.jstor.org/stable/4408255.

Cernea, M.M. (2000). Risks, safeguards and reconstruction: A model for population displacement and resettlement. En: M.M. Cernea y C. McDowell (Eds.), Risks and reconstruction: Experiences of resettlers and refugees (pp. 11-55). Washington, DC: The World Bank.

Cernea, M.M. (2003). For a new economics of resettlement: a sociological critique of the compensation principle. International Social Science Journal, 55(175), 37-45. https://doi. org/10.1111/1468-2451.5501004.

Colson, E. (1971). The social consequences of resettlement: The impact of the Kariba resettlement upon the Gwembe Tonga. Manchester: Manchester University Press.

Crecimiento urbano y acceso a oportunidades: un desafío para América Latina. (2017). Bogotá: CAF.

Crisp, J., Morris, T., y Refstie, H. (2012). Displacement in urban areas: new challenges, new partnerships. Disasters, 36(S1), S23-S42. https://doi. org/10.1111/j.1467-7717.2012.01284.x. 
Dash, S. (2008). Resettlement and rehabilitation in Orissa: A study of the upper Indravati hydro-electric project Satya. Social Change, 38(4), 661-688. https://doi.org/10.1177/004908570803800406.

Day, J. (2013). Effects of involuntary residential relocation on household satisfaction in Shanghai, China. Urban Policy and Research, 31(1), 93-117. https://doi.org/10.1080/08111146.2012.757736.

De Wet, C.J. (2006). Development-induced displacement: Problems, policies, and people. Oxford: Berghahn.

Devitt, P., y Hitchcock. R. (2010). Who drives resettlement? The case of Lesotho's Mohale Dam. African Study Monographs, 31(2), 57-106.

Driant, J.C., y Riofrío, G. (1987). ¿Qué vivienda han construido? Nuevos problemas en viejas barriadas. Lima: IFEA.

Instituto Nacional de Estadística e Informática. (2007). Censos nacionales 2007: XI de población y VI de vivienda. Lima: Autor.

Instituto Nacional de Estadística e Informática. (2014). Una mirada a Lima metropolitana. Lima: Autor.

Jiang, Y., Waley, P.T., y Gonzalez, S. (2018). 'Nice apartments, no jobs': How former villagers experienced displacement and resettlement in the western suburbs of Shanghai. Urban Studies, 55(14), 3202 - 3217. https://doi. org/10.1177/0042098017740246.

Koenig, D. (2002). Toward local development and mitigating impoverishment in development-induced displacement and resettlement. Oxford: University of Oxford.
Li, S., y Song, S. (2009). Redevelopment, displacement, housing conditions, and residential satisfaction: a study of Shanghai. Environment and Planning A: Economy and Space, 41(5), 1090-1108. https://doi. org/10.1068/a4168.

Maxwell, J. (2016). Expanding the history and range of mixed methods research. Journal of Mixed Methods Research, 10(1), 1-16. https://doi. org/10.1177/1558689815571132.

Mejía, M.C. (1999). Economic dimensions of urban resettlement: Experiences from Latin America. En M. M. Cernea (Ed.), The economics of involuntary resettlement: Questions and challenges (pp. 147188). Washington, D.C.: The World Bank.

Mejía-Escalante, M. (2012). Habitabilidad en la vivienda social en edificios para población reasentada. El caso de Medellín, Colombia. EURE, 38(114), 203-227. https://doi.org/10.4067/ S0250-71612012000200008.

Moser, C. (1998). The asset vulnerability framework: Reassessing urban poverty reduction strategies. World Development, 26(1), 1-19. https://doi. org/10.1016/S0305-750X(97)10015-8.

Necochea, A. (1987). Los allegados: una estrategia de supervivencia solidaria en vivienda. EURE, 13(39-40), 85-99. Recuperado de http://www. eure.cl/index.php/eure/article/view/1005.

Oliver-Smith, A. (2006). Displacement, resistance and the critique of development: from the grassroots to the global. En: C. J. De Wet (Ed.) Developmentinduced displacement: Problems, policies, and people. Oxford: Berghahn. 
Programa de reubicación de viviendas. (2017). Recuperado de http://www.lamsac.com.pe/sostenibilidad/programa-de-reubicacion-de-viviendas/ casos-de-exito-de-reubicacion.

Rakodi, C. (1999). A capital assets framework for analysing household livelihood strategies: Implications for policy. Development Policy Review, 17(3), 315342. https://doi.org/10.1111/1467-7679.00090.

Rakodi, C., y Lloyd-Jones, T. (2002). Urban livelihoods: A people-centred approach to reducing poverty. Londres: Earthscan.

Ramírez-Corzo, D., y Riofrío, G. (2006). Formalización de la propiedad y mejoramiento de barrios: bien legal, bien marginal. Lima: DESCO.

Riofrío, G. (2004). Pobreza y desarrollo urbano en el Perú. En: Las ciudades en el Perú (pp. 71-112). Lima: DESCO.

Robinson, C. (2003). Risks and rights: The causes, consequences, and challenges of development-induced displacement. Washington D.C.: The Brookings Institution.

Roquet, V., Bornholdt, L., Sirker, K., y Lukic, J. (2017). Urban land acquisition and involuntary resettlement linking innovation and local benefits. Washington, D.C.: The World Bank.

Santiago, S. (1977). Staying where the action is: Relocation within the city. Philippine Sociological Review, 25(1/2), 45-49. Recuperado de http://www.jstor. org/stable/43596350.

Sepúlveda, 0. (1986). El espacio en la vivienda social y calidad de vida. Revista INVI, 1(2), 10-34.
Recuperado de http://www.revistainvi.uchile.cl/ index.php/INVI/article/view/78.

Siegel, P.B. (2005). Using an asset-based approach to identify drivers of sustainable rural growth and poverty reduction in Central America: A conceptual framework. Washington, D.C.: The World Bank.

Strauch, L., Takano, G., y Hordijk, M. (2015). Mixed-use spaces and mixed social responses: Popular resistance to a megaproject in central Lima, Peru. Habitat International, 45(3), 177-184. https://doi. org/10.1016/j.habitatint.2014.02.005.

Takano, G. (2017). Public-private partnerships as rent-seeking opportunities: A case study on an unsolicited proposal in Lima, Peru. Utilities Policy, 48, 184-194. https://doi.org/10.1016/j. jup.2017.08.005.

Terminski, B. (2013). Development-induced displacement and resettlement: Social problem and human rights issue. Ginebra: University of Geneva.

Torrado, S. (1982). El enfoque de las estrategias familiares de vida en América Latina. Orientaciones teórico-metodológicas. En Familia y diferenciación social. Cuestiones de método. Buenos Aires: EUDEBA.

Vaughan, M. (1983). Which family?: Problems in the reconstruction of the history of the family as an economic and cultural unit. The Journal of African History, 24(2), 275-283. https://doi.org/10.1017/ S0021853700021988.

Walsh Perú S.A. (2011). Estudio de impacto ambiental vía expresa línea amarilla. Lima: Walsh Perú S.A. 
Wilmsen, B., y Van Hulten, A. (2017). Following resettled people over time: the value of longitudinal data collection for understanding the livelihood impacts of the Three Gorges Dam, China. Impact Assessment and Project Appraisal, 35(1), 94-105. https://doi.org/10.1080/14615517.2016.1271542.

Wheelock, J., y Oughton, E. (1996). The household as a focus for research. Journal of Economic Issues, 30(1), 143-159. https://doi.org/10.1080/0021362 4.1996.1150577 